\title{
Stat3 regulates desmoglein 3 transcription in epithelial keratinocytes
}

\author{
Xuming Mao, Michael Jeffrey T. Cho, Christoph T. Ellebrecht, Eric M. Mukherjee, \\ and Aimee S. Payne \\ Department of Dermatology, University of Pennsylvania, Philadelphia, Pennsylvania, USA.
}

\begin{abstract}
Pemphigus vulgaris (PV) is an epithelial blistering disease caused by autoantibodies to the desmosomal cadherin desmoglein 3 (DSG3). Glucocorticoids improve disease within days by increasing DSC3 gene transcription, although the mechanism for this observation remains unknown. Here, we show that DSC3 transcription in keratinocytes is regulated by Stat3. Treatment of primary human keratinocytes (PHKs) with hydrocortisone or rapamycin, but not the P38 MAPK inhibitor SB202190, significantly increases DSC3 mRNA and protein expression and correspondingly reduces phospho-S727 Stat3. Stat3 inhibition or shRNA-knockdown also significantly increases DSC3 mRNA and protein levels. Hydrocortisone- or rapamycin-treated PHKs demonstrate increased number and length of desmosomes by electron microscopy and are resistant to PV IgG-induced loss of cell adhesion, whereas constitutive activation of Stat3 in PHKs abrogates DSG3 upregulation and inhibits hydrocortisone and rapamycin's therapeutic effects. Topical hydrocortisone, rapamycin, or Stat3 inhibitor XVIII prevents autoantibody-induced blistering in the PV passive transfer mouse model, correlating with increased epidermal DSC3 expression and decreased phospho-S727 Stat3. Our data indicate that glucocorticoids and rapamycin upregulate DSC3 transcription through inhibition of Stat3. These studies explain how glucocorticoids rapidly improve pemphigus and may also offer novel insights into the physiologic and pathophysiologic regulation of desmosomal cadherin expression in normal epidermis and epithelial carcinomas.
\end{abstract}

Conflict of interest: The authors have declared that no conflict of interest exists.

Submitted: December 21, 2016 Accepted: March 23, 2017 Published: May 4, 2017

Reference information: JCI Insight. 2017;2(9):e92253. https:// doi.org/10.1172/jici.insight.92253.

\section{Introduction}

Pemphigus vulgaris (PV) is a life-threatening autoimmune disease characterized by suprabasal blistering of the skin and mucous membranes. PV autoantibodies target the keratinocyte adhesion protein desmoglein 3 (DSG3), causing DSG3 endocytosis and desmosomal DSG3 depletion (1-3), resulting in the loss of intercellular adhesion (acantholysis). Most PV therapies suppress the immune system to decrease antibody production. Glucocorticoids are first-line therapy in PV because, in addition to their immunosuppressive function, they upregulate DSG3 gene transcription (4), which rapidly counteracts the PV IgG-induced loss of DSG3 from desmosomes. However, the mechanism by which glucocorticoids upregulate DSG3 transcription remains poorly defined.

Rapamycin, an mTOR inhibitor, was reported to induce rapid remission of PV (5), leading us to speculate that rapamycin also upregulates DSG3 transcription. Although the DSG3 promoter has been cloned (6), no prior studies have identified transcription factors that bind the DSG3 promoter. In this work, we aimed to identify the molecular mechanisms regulating DSG3 gene expression. We find that glucocorticoids and rapamycin upregulate DSG3 transcription through inhibition of the transcription factor Stat3. These findings have biologic significance not only for the mechanism of rapid disease control in PV, but also for the physiologic regulation of DSG3 expression in the epidermis and its dysregulation in various disease states.

\section{Results}

Glucocorticoids and rapamycin promote DSG3 gene transcription in keratinocytes, resulting in increased desmosome number and length. To determine whether rapamycin, like glucocorticoids, increases DSG3 expression at the transcriptional level, we performed quantitative PCR (qPCR) using DSG3-specific primers on primary human keratinocyte (PHK) cDNA. Treatment of PHKs with $25 \mathrm{nM}$ rapamycin and 100 $\mu \mathrm{g} / \mathrm{ml}$ hydrocortisone results in a 2.1-fold and 1.6-fold increase in DSG3 mRNA expression compared with DMSO control $(P<0.05)$, whereas the p38 MAPK inhibitor SB202190 (SB) $(5 \mu \mathrm{M})$ does not 
A

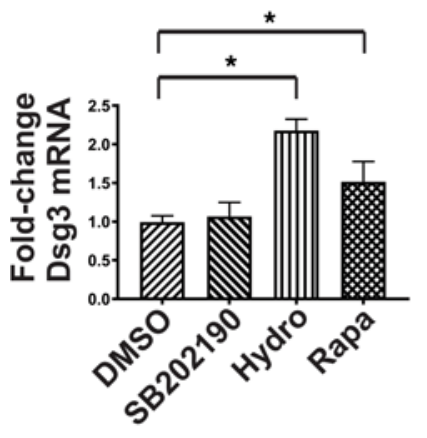

D

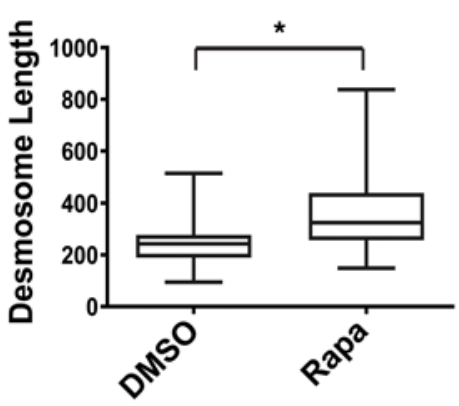

E

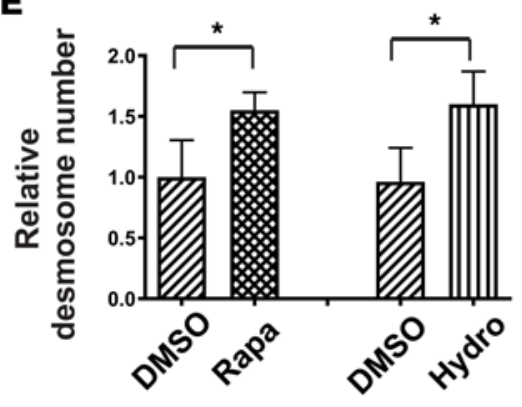

B
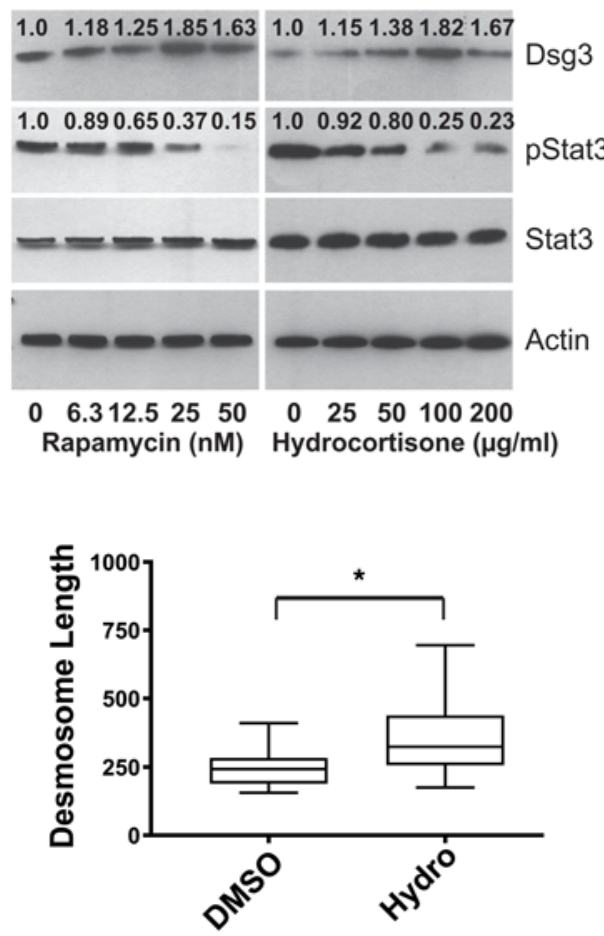

$\mathbf{F}$

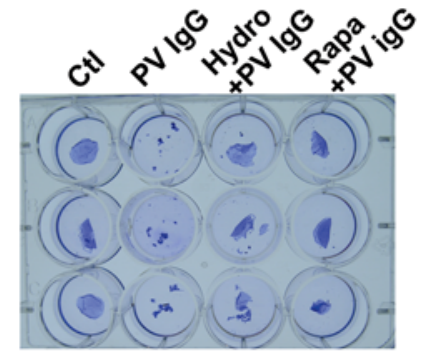

C

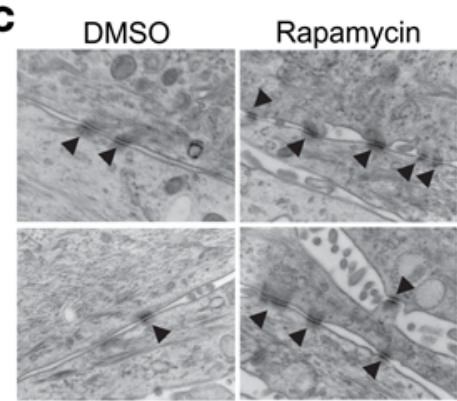

DMSO

Hydrocortisone

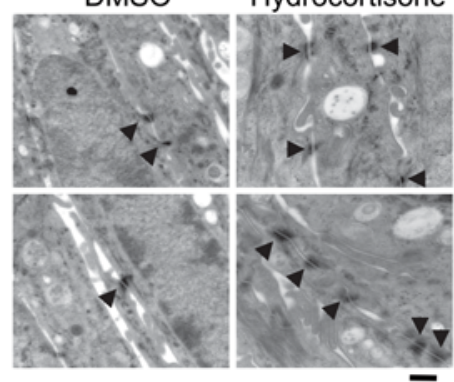

G

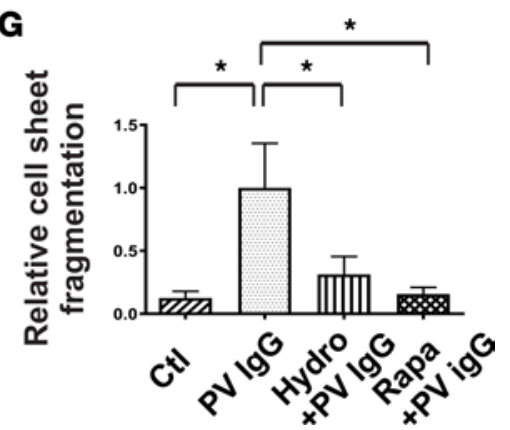

Figure 1. Glucocorticoids and rapamycin increase desmoglein 3 (DSG3) transcription and bolster desmosomal adhesion through increased desmosome length and number. (A) qPCR indicates a significant increase in DSC3 transcription after treatment of primary human keratinocytes (PHKs) with hydrocortisone (Hydro) and rapamycin (Rapa) but not the p38 inhibitor SB202190. ${ }^{*} P<0.05$ (one-way ANOVA). (B) Hydrocortisone and rapamycin cause a dose-dependent increase in DSG3 protein levels and a decrease in phospho-S727 Stat3 (pStat3), shown by immunoblot. Results are representative of 2-3 different experiments. Electron microscopic analysis of PHKs (C, scale bar: $500 \mathrm{~nm}$ ) indicates increased desmosome length (D) and number (E) after treatment with hydrocortisone and rapamycin ( ${ }^{*} P<0.05$, Wilcoxon rank-sum and 2 tailed Student's $t$ test, respectively), corresponding to significantly decreased PHK cell sheet fragmentation by PV IgG (F and $\mathbf{G}$, results are representative of 3 different experiments). ${ }^{*} P<0.05$ (one-way ANOVA).

significantly affect DSG3 expression (Figure 1A). Both hydrocortisone $(0-100 \mu \mathrm{g} / \mathrm{ml})$ and rapamycin $(0-25 \mathrm{nM})$ cause dose-dependent increases in DSG3 protein levels, concomitant with a dose-dependent decrease in phopho-S727 Stat3 (pStat3), but slight decreases in Dsg3 protein levels were observed at higher hydrocortisone and rapamycin doses (Figure 1B).

Electron microscopy (Figure 1C) revealed that rapamycin and hydrocortisone significantly increase average desmosome length in PHKs to $324 \mathrm{~nm}$ and $340 \mathrm{~nm}$, respectively, compared with controls (213-243 nm) (Figure 1D) and also cause a 1.6-fold increase in relative desmosome number (Figure 1E) $(P<0.05)$. Consistent with these observations, hydrocortisone and rapamycin protect $\mathrm{PHKs}$ against acantholysis induced by PV serum IgG (Figure 1F), evidenced by significantly reduced cell sheet fragmentation (Figure 1G).

Glucocorticoids and rapamycin increase DSG3 transcription in a Stat3-dependent manner. Analysis of published ChIP-seq data revealed several transcription factors that demonstrate high signal strength for occupancy at the DSG3 promoter (Figure 2). We focused on Stat3, as it is known to be a direct downstream target of mTOR (7) and also synergizes with the glucocorticoid receptor for transcription regulation (8). 


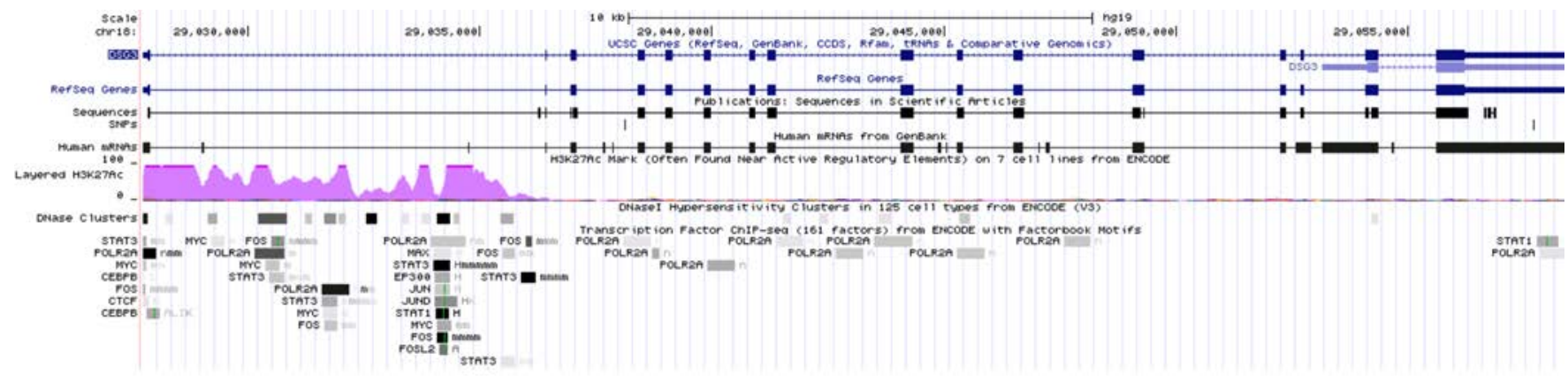

Figure 2. Bioinformatic analysis of the desmoglein 3 (DSC3) promoter identifies Stat3 as a putative DSG3 transcription factor. ChIP-seq data from the Encyclopedia of DNA Elements (ENCODE) database are shown.

Treatment of PHKs with $25 \mu \mathrm{M}$ Stat3 inhibitor XVIII causes a 3.5-fold increase in DSG3 expression (Fig ure 3A) and a dose-dependent increase in DSG3 protein level (Figure 3B). Stat3 inhibition in PHKs was also associated with increased protein levels of the desmosomal adhesion molecules DSG1 and DSG2, as well as the adherens junction molecule E-cadherin, whereas levels of pan-keratin and desmocollin 3 (DSC3) were unchanged to decreased (Supplemental Figure 1; supplemental material available online with this article; https://doi.org/10.1172/jci.insight.92253DS1). Stat3 inhibition correspondingly prevents PV IgG-induced keratinocyte cell sheet fragmentation (Figure 3, C and D). Confirming the specificity of the inhibitor studies, shRNA-mediated knockdown of Stat3 increases DSG3 protein levels in PHKs (Figure 3E). To determine whether Stat3 occupies the DSG3 promoter in PHKs, we performed ChIP-PCR. DSG3 promoter amplification occurs after chromatin immunoprecipitation with antibodies specific for mRNA polymerase and Stat3, but not Stat1 or antibody isotype controls (Figure 3F).

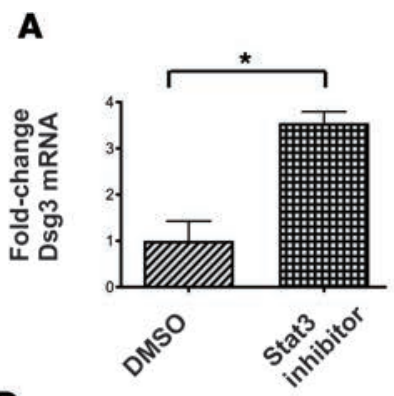

A

D

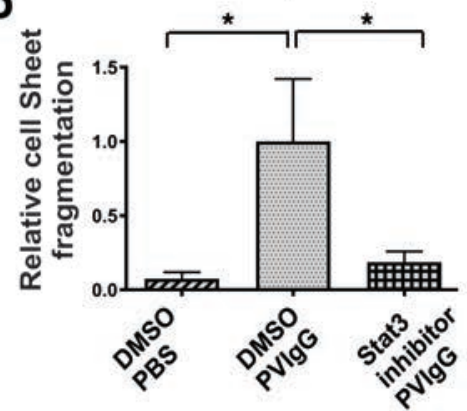

E

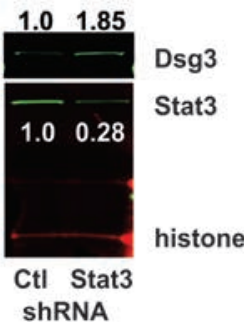

B

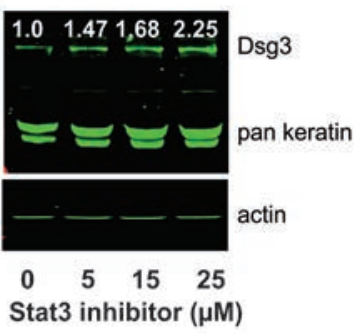

F

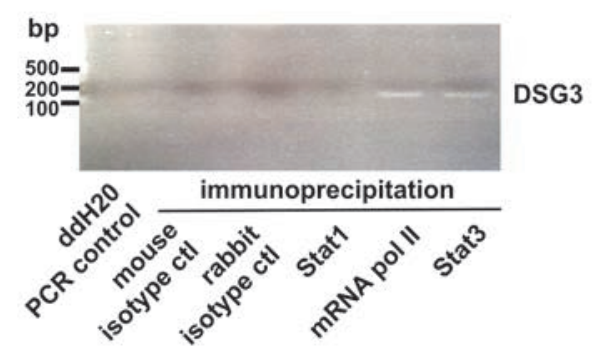

G

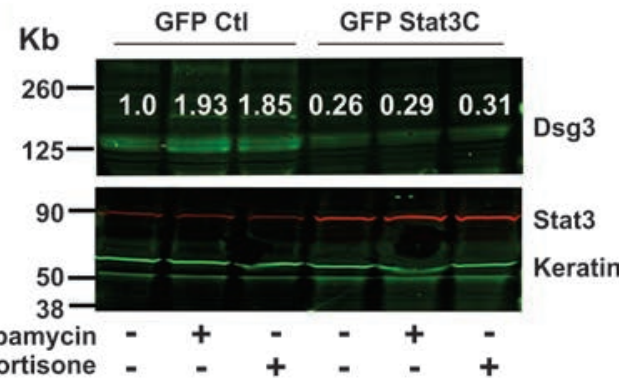

C

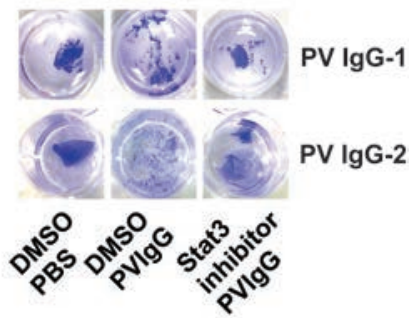

SG3 tion prevents pemphigus vulgaris ( $\mathrm{PV}$ ) IgG-induced loss of keratinocyte adhesion. Two PV IgG samples shown; results are representative of 3 and 2 different experiments, respectively. (D) Stat3 inhibition significantly reduces $\mathrm{PHK}$ cell sheet fragmentation (C, PV IgG-1). ${ }^{*} P<$ 0.05 (one-way ANOVA). (E) Stat3 shRNA knockdown increases DSG3 protein levels. Results are representative of 2 different experiments. (F) ChIP-PCR indicates that Stat3 occupies the DSC3 promoter in PHKs. Results are representative of 2 different experiments. (G) Expression of constitutively activated Stat3 (Stat3C) reduces basal DSG3 protein expression levels and blocks the hydrocortisone- and rapamycin-induced upregulation of DSG3. Results are representative of 3 different experiments. 

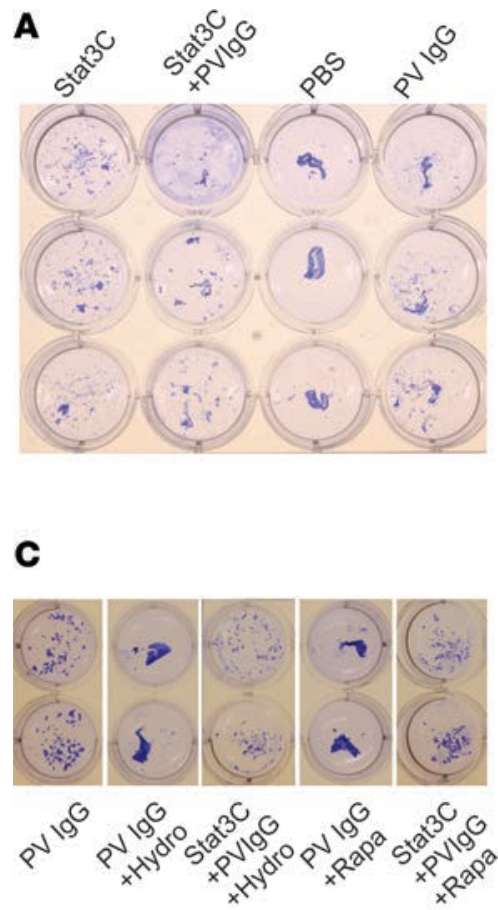

B

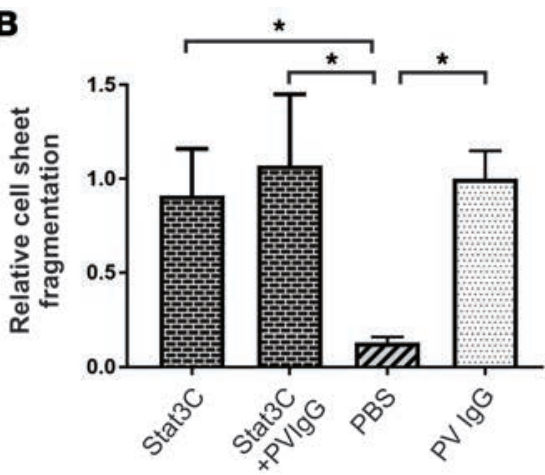

D

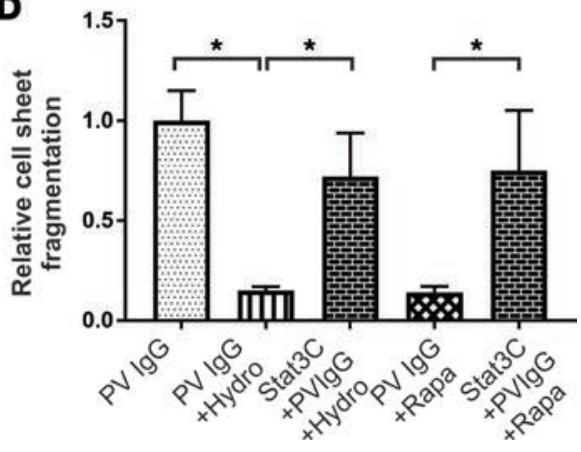

Figure 4. Constitutive activation of Stat3 (Stat3C) inhibits the protective therapeutic effects of hydrocortisone and rapamycin on pemphigus vulgaris (PV) IgG-induced loss of cell adhesion. (A) Constitutive activation of Stat3 (Stat3C) in primary human keratinocytes (PHKs) reduces basal keratinocyte adhesion. Results are representative of 3 different experiments. (B) Constitutive activation of Stat3 as shown in $\mathbf{A}$ significantly reduces PHK adhesion; no significant increase in cell sheet fragmentation is observed with addition of PV IgG. ${ }^{*} P<0.05$ (one-way ANOVA). (C) Constitutive activation of Stat3 in PHKs inhibits the protective effects of hydrocortisone (Hydro) and rapamycin (Rapa) against PV IgG. Results are representative of 2 different experiments. (D) Quantitation of C. ${ }^{*} P<0.05$ (one-way ANOVA).

We next investigated whether Stat 3 activation can block the glucocorticoid- and rapamycin-induced increase in DSG3 protein levels. In control GFP-expressing PHKs, both rapamycin and hydrocortisone cause a 1.9-fold increase in DSG3 protein level (Figure 3G). In contrast, constitutively active Stat3 (Stat3C) (9) not only abrogates the rapamycin- and hydrocortisone-induced upregulation of DSG3, but also reduces basal levels of DSG3 protein in PHKs by 74\%, indicating that Stat3 governs DSG3 expression in keratinocytes.

Constitutive activation of Stat 3 inhibits the protective therapeutic effects of hydrocortisone and rapamycin on PV IgG-induced loss of keratinocyte adhesion. We next determined whether constitutive activation of Stat3 in PHKs has functional consequences on cell adhesion. Preliminary experiments indicated that PHK transduction efficiency with lentiviral GFP particles was approximately $75 \%$ (data not shown). Consistent with the reduction in basal Dsg3 expression by Stat3C observed in Figure 3G, Stat3C caused increased cell sheet fragmentation even in the absence of PV IgG, indicating a weakened adhesive state. Addition of PV IgG to Stat3C-expressing PHKs caused a minimal increase in total cell sheet fragmentation (Figure 4, A and B). Additionally, consistent with the lack of Dsg3 upregulation observed in Stat3C-expressing PHKs after hydrocortisone and rapamycin treatment, constitutive activation of Stat3 inhibits the protective effects of hydrocortisone and rapamycin on PV IgG-induced loss of cell adhesion in PHKs (Figure 4, C and D).

Topical Stat3 inhibition prevents PV IgG and anti-DSG3 mAb-induced skin blistering in a PV mouse model, phenocopying the protective effects of topical glucocorticoids and rapamycin. Application of topical hydrocortisone and rapamycin daily for 3 days on the back skin of mice causes increased DSG3 protein levels in epidermis, concomitant with decreased phospho-S727 Stat3 (Figure 5A). Topical hydrocortisone and rapamycin protect against skin blisters induced by passive transfer of PV IgG by both gross and histologic analysis (Figure $5, \mathrm{~B}$ and $\mathrm{C}$ ), resulting in a significant decrease in the extent of skin blistering (Figure 5D) despite equivalent binding of PV IgG to the keratinocyte cell surface by direct immunofluorescence analysis (Figure $5 \mathrm{E}$ ).

Similarly, topical application of Stat3 inhibitor XVIII decreases phospho-S727 Stat3 in mouse epidermis, concomitant with an upregulation of DSG3 protein expression (Figure 6A). Topical Stat3 inhibition significantly decreases PV IgG-induced blistering despite equivalent binding of PV IgG to mouse skin (Figure 6, B-D). Topical Stat3 inhibitor also protects from pathogenic PV anti-DSG3/1 $\mathrm{mAb}$-induced (Px43-induced) skin blisters (Supplemental Figure 2).

\section{Discussion}

Using targeted bioinformatic analysis, ChIP-PCR, qPCR, and functional studies in primary keratinocyte culture and in mouse models, our studies have identified the first transcription factor that binds to the promoter of DSG3 to regulate its transcription in keratinocytes. Our studies indicate that Stat 3 inhibition 
A

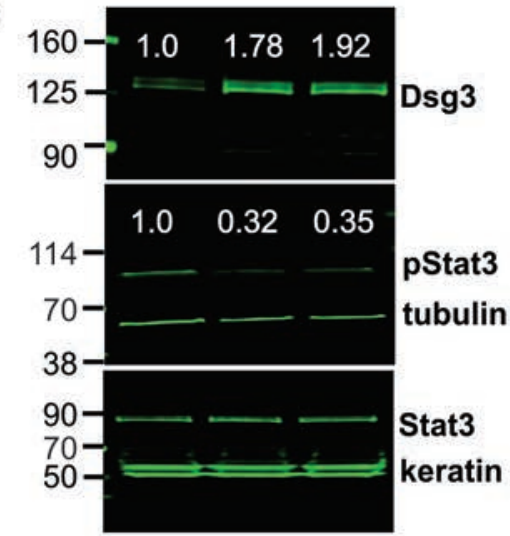

B

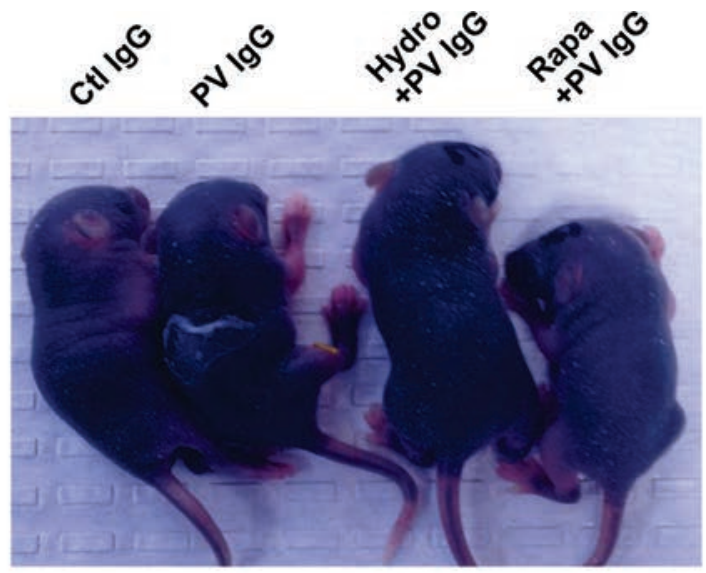

\section{$\begin{array}{rlll}\text { Hydro } & - & + & - \\ \text { Rapa } & - & - & +\end{array}$}

C

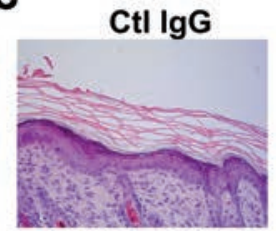

Hydro+PVlgG

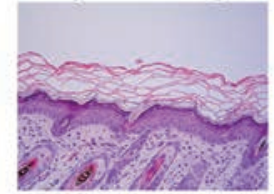

PV IgG

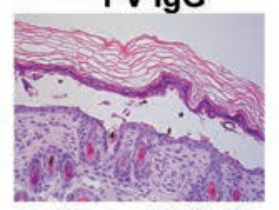

Rapa+PVIgG

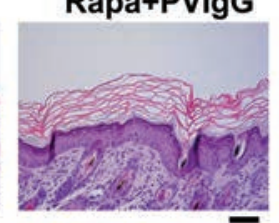

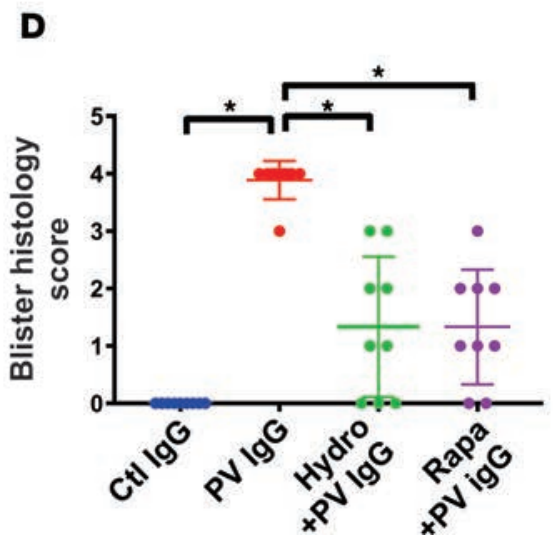

E

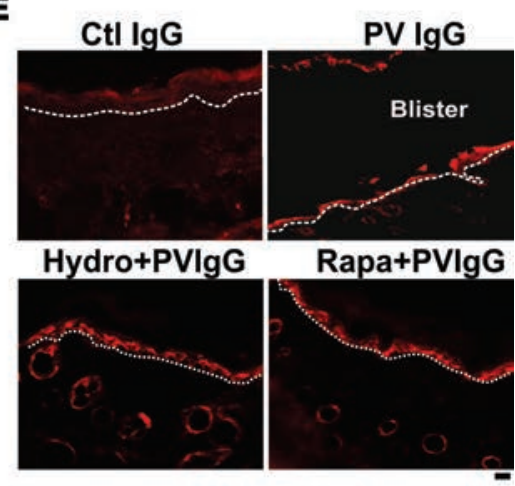

Figure 5. Topical hydrocortisone and rapamycin inhibit Stat3 and increase desmoglein 3 (DSG3) protein expression in vivo, resulting in protection from pemphigus vulgaris (PV) IgG-induced skin blistering. (A) Immunoblot analysis of mouse skin after topical application of hydrocortisone and rapamycin indicates increased DSG3 protein levels and decrease in phospho-S727 Stat3 (pStat3). Results are representative of 2 different experiments. (B) Topical hydrocortisone and rapamycin prevent gross and (C) histologic blisters after passive transfer of PV IgG to neonatal mice. Scale bar: $100 \mu \mathrm{m}$. Results are representative of 2 different experiments. (D) Skin blister scores are significantly decreased in hydrocortisone- and rapamycin-treated mice compared with control mice. ${ }^{*} P<0.05$ (Kruskal-Wallis test). (E) Direct immunofluorescence analysis of mouse skin indicates equal binding of PV IgC to the keratinocyte cell surface in all mice. Scale bar: $20 \mu \mathrm{m}$.

by glucocorticoids and rapamycin is sufficient for Dsg3 upregulation and that this protective effect on cell adhesion can be overridden through constitutive activation of Stat3. However, these findings do not exclude that other signaling pathways may contribute to the regulation of DSG3 transcription or that other cellular mechanisms regulating DSG3 protein levels at the posttranscriptional level could additionally be affected by Stat3 inhibition.

Physiologic DSG3 expression is limited to stratified squamous epithelia (10) and thymic epithelia, the latter of which is controlled by the autoimmune regulator transcription factor AIRE (11). An Oct-like motif between -200 and -170 in the DSG3 promoter was previously implicated in conferring basal DSG3 transcription in human keratinocytes (6). However, this sequence region - while not a perfect match for an Oct domain - exactly matches the C/EBP $\beta$ consensus site 5'-T[TG]NNGNAA[TG]-3' (12). Thus, we predict that C/EBP $\beta$ promotes basal DSG3 transcription in epidermal keratinocytes and that Stat3 activation can reduce DSG3 expression both in keratinocytes and other cell types.

Stat 3 binds to the palindromic sequence $5^{\prime}$-TTCNNNGAA-3' $(13,14)$, but the mere presence of a Stat3 binding site does not predict Stat3 promoter occupancy or the transcriptional response to Stat3 binding. Computational analysis of ChIP-seq data has revealed that the majority of Stat3 promoter occupancy sites are cell type-specific. Myriad factors affect Stat3's transcriptional regulatory ability, including 
A

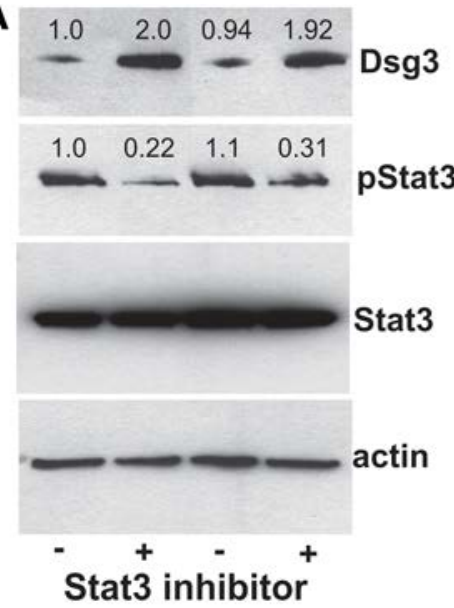

C

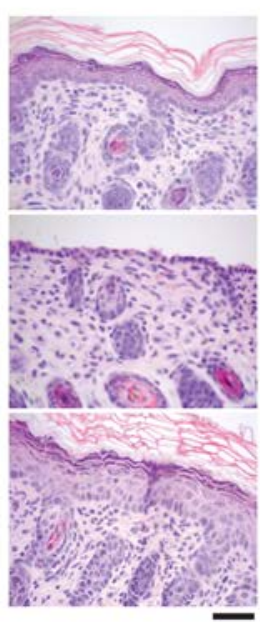

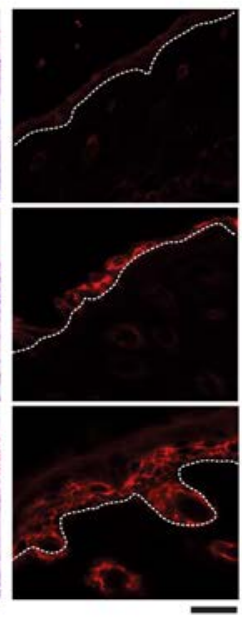

\section{B}
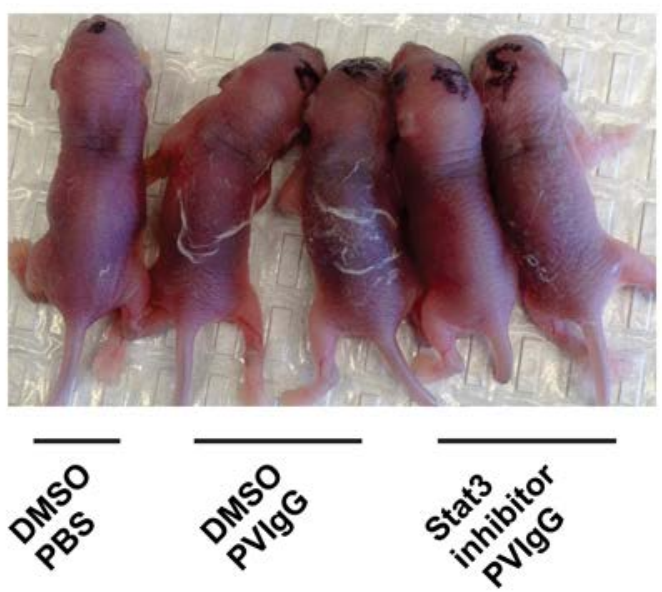

D

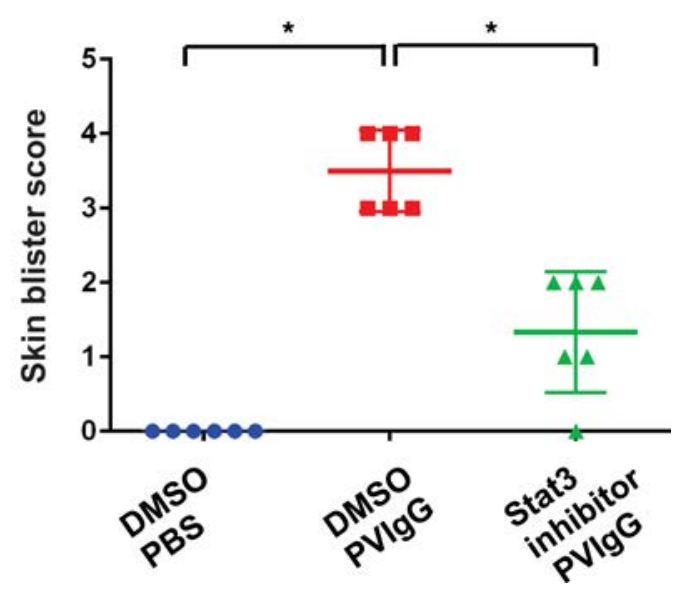

Figure 6. Stat3 inhibition prevents skin blistering in a pemphigus vulgaris (PV) mouse model. (A) Topical Stat3 inhibition results in increased desmoglein 3 (DSG3) protein levels and decreased phospho-S727 Stat3 (pStat3) in mouse epidermis. Results are representative of 2 different experiments. Stat3 inhibitor XVIII abrogates skin blisters induced by passive transfer of PV IgG at the gross (B) and histologic levels (C, left panels), despite equivalent binding of PV IgG to the keratinocyte cell surface (C, right panels). Scale bars: $100 \mu \mathrm{m}$. Results are representative of 2 different experiments. (D) A significant decrease in skin blister scores is observed in PV model mice treated with topical Stat3 inhibitors. ${ }^{*} P<0.05$ (Kruskal-Wallis test).

phosphorylation, acetylation, epigenetic influences on DNA binding site accessibility, and interaction with different protein cofactors that modulate DNA binding site preferences (15). Tyrosine phosphorylation of Stat3 by Janus kinases promotes Stat3 dimerization, nuclear translocation, and DNA binding. Serine kinases play a more nuanced role in regulating Stat3 due to direct Stat3 activation through S727 phosphorylation (16) by kinases such as mTOR (7) and others, which also have the potential for competing inhibitory effects from parallel downregulation of Stat 3 tyrosine phosphorylation pathways (17). Stat 3 also interacts with the histone acetyltransferase p300, which acetylates Stat 3 on lysine 685 to stabilize Stat3 dimerization (18). p300 binding causes epigenetic remodeling through histone acetylation, which promotes local gene expression, or recruitment of DNA methyltransferase 1 to acetylated Stat3 to downregulate transcription (19). Our studies suggest that there may be a dose optimum for Dsg3 upregulation by glucocorticoids and rapamycin, underscoring the complexity of the regulatory pathways. Future studies will be necessary to determine the network of Stat3 cofactors that collectively regulate DSG3 expression.

Loss of Stat3 is embryonic lethal (20). Keratinocyte-specific deficiency of Stat3 leads to impaired keratinocyte migration (21) and resistance to epithelial carcinogenesis (22). Conversely, constitutive activation of Stat3 in skin (K5.Stat3C) induces psoriasis-like skin inflammation (23) and enhanced initiation, promotion, and invasion of squamous cell carcinoma (SCC) (24). Although no epithelial 
blistering was observed in the K5.Stat3C mouse model, we speculate that acantholytic SCCs - which show loss of intercellular adhesion, loss of DSG3 staining, and potentially poorer prognosis $(25,26)$ - may demonstrate Stat3 activation. Poor prognosis in head and neck SCC is also associated with loss of DSG3 expression (27), suggesting that these poorly differentiated tumors may also be more likely to demonstrate Stat3 activation. Altered expression of desmosomal cadherins has also been noted in colorectal carcinoma, where switching from desmocollin 2 to desmocollin 1 and -3 is related to oncogenesis (28), although the cause-effect relationship remains uncertain. Our studies, to our knowledge, provide a novel framework to better understand desmosomal cadherins as biomarkers in epithelial carcinomas and further investigate transitions in desmosomal cadherin expression as a result of altered transcriptional signaling pathways.

Finally, our data have direct medical relevance to how glucocorticoids work rapidly to control disease in PV by upregulating the expression of the target autoantigen to combat its destruction by antiDSG3 antibodies. Stat3 inhibition and rapamycin would similarly be predicted to have rapid therapeutic effect in PV, thus providing an alternative strategy to corticosteroids as first-line treatment. Consistent with this model, 2 prior cases of PV treated with systemic rapamycin reported rapid disease improvement $(5,29)$. However, a case series of 3 patients with oral PV noted no effect of topical rapamycin (30). The variability of rapamycin's reported efficacy in PV may be due to suboptimal topical dosing or limits on topical versus systemic efficacy, as is also observed with the use of topical versus systemic glucocorticoids in PV, particularly in more severe cases with high titer autoantibodies. Nevertheless, our data suggest that topical Stat3 inhibition may provide a valuable therapy for control of PV blistering (Figure 3). Glucocorticoids were previously shown to upregulate expression of not only DSG3, but also DSG1 and E-cadherin (4), which may also explain their therapeutic efficacy in the related autoimmune disorder pemphigus foliaceus, characterized by autoantibodies to the homologous desmosomal cadherin DSG1. Similarly, Stat3 inhibition in PHKs causes upregulation of other adhesion molecules — including DSG1, DSG2, and E-cadherin (Supplemental Figure 1) - suggesting that Stat3 inhibition is likely to have a therapeutic effect for pemphigus foliaceus in addition to PV.

Phase I clinical trials in cancer have provided insight into the potential efficacy and toxicities of Stat3 inhibition as a therapeutic approach. Small molecule Stat3 inhibitor OPB-51602 has shown therapeutic promise, with tolerable gastrointestinal and peripheral neuropathy side effects (31). A Stat3 antisense oligonucleotide AZD9150 showed preliminary efficacy, with side effects of thrombocytopenia attributed to Stat3's role in megakaryopoiesis and transaminase elevation likely secondary to the delivery system (32). An intratumorally injected Stat3 oligonucletide decoy, which sequesters Stat 3 to prevent it from binding its genomic targets, showed mild therapeutic responses with no dose-limiting toxicity (33), suggesting that local application may provide targeted efficacy without systemic side effects.

In summary, this report, to our knowledge, identifies Stat3 as a novel regulator of DSG3 expression in human keratinocytes. Future studies will help to elucidate the physiologic patterns of desmosomal cadherin expression in the epidermis and their aberrant expression in different disease states, and they may ultimately lead to novel adjunctive strategies for pemphigus therapy.

\section{Methods}

Antibodies and reagents. Antibodies against Stat3 (catalog 4904), phospho-S727 Stat3 (catalog 9134), and actin were purchased from Cell Signaling Technology ; DSG3 antibody (5G11, catalog 32-6300) were purchased from Thermo Scientific; antibodies against DSP (catalog A303-355A-T), Stat1 (catalog A302-752A-T), and mRNA polymerase (catalog A300-654A-T) were purchased from Bethyl Laboratories; antibody against plakoglobin (catalog MAB2038) was purchased from Chemicon; and antibodies against histone H3 (catalog sc-8654) and DSG2 (catalog sc-80663) were purchased from Santa Cruz Biotechnology Inc.

Rapamycin and SB were purchased from Cell Signaling Technology; Stat3 inhibitor XVIII (BP-1-102) was purchased from Calbiochem; and hydrocortisone was purchased from Sigma-Aldrich. Stat3C lentiviral plasmid (EF.STAT3C.Ubc.GFP, catalog 24983) and control GFP plasmid (catalog 52581) were purchased from Addgene.

PV IgG-1 was purified using Melon Gel (Thermo Fisher Scientific) from the serum of a patient with severe mucocutaneous PV, who demonstrated a Dsg3 index value of 191 and Dsg1 index value of 240. PV IgG-2 was similarly isolated from a patient with severe mucocutaneous PV, demonstrating a Dsg3 index value of 183 and Dsg1 index value of 158. All studies utilized PV IgG-1 unless otherwise indicated. 
Cell culture and treatments. PHKs isolated from neonatal foreskin were obtained from the Penn Skin Biology and Diseases Resource-based Center and were cultured in defined keratinocyte serum-free media (DK-SFM) (Invitrogen) containing $0.07 \mathrm{mM} \mathrm{CaCl}_{2}$ and penicillin/streptomycin (Invitrogen) and maintained in a humidified incubator with $5 \% \mathrm{CO}_{2}$ at $37^{\circ} \mathrm{C}$. For all in vitro experiments, concentrations of 25 $\mathrm{nM}$ rapamycin, $100 \mu \mathrm{g} / \mathrm{ml}$ hydrocortisone, $5 \mu \mathrm{M}$ SB202190, and $25 \mu \mathrm{M}$ Stat3 inhibitor XVIII were tested, unless otherwise indicated.

$R N A$ purification and $q P C R$. Following treatments, total RNA was extracted from PHKs using the RNeasy Mini Kit (Qiagen). First-strand cDNA was generated by reverse transcription using the Superscript First-Strand synthesis system (Invitrogen). For quantification of DSG3 mRNA levels, the following primers were used for PCR: forward primer 5'-TTCCTGATCACATGTCGGGC-3' and reverse primer 5'-CACCAGTGAGTTTGAGGCACT-3', with SYBR Green Master Mix (Thermo Fisher Scientific) or SYBR GreenER SuperMix (Invitrogen). The expression of $\beta$-actin was used as an internal control. All qPCR reactions were carried out on the ViiA 7 Real-Time PCR system (Applied Biosystems).

Bioinformatic analysis. Putative transcription factor binding sites were identified by ChIP-seq data according to the UCSC Genome Browser using the human assembly Feb.2009 (GRCh37/hg19) at https://genome.ucsc.edu (34).

Direct immunofluorescence studies. Skin tissue samples were obtained from mice after passive transfer with PV IgG with or without topical treatments of hydrocortisone or rapamycin. Frozen skin sections were prepared and blocked in PBS containing 2\% BSA for 60 minutes, followed by incubation with primary antibodies in blocking solution for 1 hour at room temperature. After washing with PBS, cells were incubated with anti-human IgG secondary antibodies (Molecular Probes) diluted 1:200 in blocking solution for 30-60 minutes at room temperature. Binding of PV IgG was visualized with an Olympus BX61 microscope, and images were acquired using Slidebook 4.2 software (Olympus) and a Hamamatsu Orca ER camera.

Histology and IHC. Formalin-fixed, paraffin-embedded mouse skin sections were stained with $\mathrm{H} \& \mathrm{E}$ according to standard procedures. Mouse skin sections were obtained after treatments, followed by fixation in 10\% formalin in PBS. Immunohistochemical analysis was performed to evaluate kinase activation, incubating a rabbit anti-phospho-Stat3 Ser727 or phospho-mTOR antibody (Cell Signaling Technologies) (1:200) with sections overnight at $4{ }^{\circ} \mathrm{C}$, followed by peroxidase-conjugated donkey anti-rabbit IgG for 1 hour at room temperature.

Electron microscopy. PHKs were seeded onto gold-coated coverslips and grown to confluence in DK-SFM, followed by $0.4 \mathrm{mM} \mathrm{CaCl}$ for 16 hours and DMSO, hydrocortisone, or rapamycin as indicated. Cells were washed in PBS and fixed with $2.5 \%$ glutaraldehyde (Sigma-Aldrich) and 2\% paraformaldehyde (Affymetrix) in $0.1 \mathrm{M}$ sodium cacodylate buffer for 1 hour. The cells were post-fixed with $1 \%$ osmium tetroxide (Sigma-Aldrich) and dehydrated with ethanol. After infiltration, selected areas of the slide were selected for flat embedding with premade blank epoxy blocks and polymerized at $68^{\circ} \mathrm{C}$ for 48 hours. One surface semithin section was cut and stained with toluidine blue to screen the cell population, and ultrathin sections were cut parallel to the culture surface. After staining with uranyl acetate and lead citrate, the sections were examined with a FEI Tecnai-T12 transmission electron microcope operated at $80 \mathrm{kv}$. To evaluate intercellular contacts, digital images $(\times 1,100)$ were taken from multiple mesh holes with a Gatan $2 \mathrm{~K}$ digital camera using image acquisition software (DigitalMicrograph) from the same vendor. Relative desmosome density was measured as the number of desmosomes per high-powered field relative to DMSO-treated cultures.

Immunoblotting. PHKs were cultured to $90 \%-100 \%$ confluence in DK-SFM with $5 \% \mathrm{CO}_{2}$ at $37^{\circ} \mathrm{C}$ before experiments. After appropriate treatments, cells were chilled on ice and washed with ice cold PBS containing calcium and magnesium (Mediatech Inc.). The cells were collected in Laemmli sample buffer containing 5\% $\beta$-mercaptoethanol (Bio-Rad). Approximately $50 \mu \mathrm{g}$ of each sample fraction was separated by SDS-PAGE (Bio-Rad). The gels were transferred to nitrocellulose, and membranes were incubated with primary antibodies for immunoblotting according to standard procedures.

$R N A$ interference. STAT3 or control shRNA lentiviral particles (Santa Cruz Biotechnology Inc.) were used to infect PHK according to the manufacturers' protocols. Seventy-two hours after transduction, cells transduced with MK2 or control shRNA lentiviral particles were selected with media containing $5 \mu \mathrm{g} / \mathrm{ml}$ puromycin (Sigma-Aldrich). Expression levels of Stat3 and its effect on DSG3 in transduced PHK cells were examined after transduction with immunoblotting. 
Dispase assay. PHKs were seeded into 12-well tissue culture plates in DK-SFM media (Thermo Fisher Scientific) with DMSO, $100 \mu \mathrm{g} / \mathrm{ml}$ hydrocortisone, $25 \mathrm{nM}$ rapamycin, or $25 \mu \mathrm{M}$ Stat 3 inhibitor pretreatment as indicated. Confluent wells were incubated with PV IgG diluted in DK-SFM media supplemented with $1.2 \mathrm{mM} \mathrm{CaCl} 2$ (Sigma-Aldrich) for 6 hours at $37^{\circ} \mathrm{C}$. Wells were washed with PBS containing calcium and magnesium and incubated with dispase (Roche Diagnostics) at $37^{\circ} \mathrm{C}$ for 30 minutes. Cell monolayers were washed with PBS containing calcium and magnesium and subjected to 5 rounds of pipetting with a $1 \mathrm{ml}$ pipettor. Cell monolayers were stained with $0.04 \%$ crystal violet (Sigma-Aldrich), and cell fragments were counted using ImageJ (NIH).

Chromatin immunoprecipitation assay. Chromatin immunoprecipitations to detect Stat3 binding was performed using the MAGnify Chromatin Immunoprecipitation System (Invitrogen) according to the manufacturer's protocol. Briefly, PHKs were fixed in 1\% formaldhyde in DK-SFM for 10 minutes at room temperature following treatments. The cells were harvested in lysis buffer containing protease inhibitors, followed by sonication to obtain 200-500 bp genomic DNA fragments. Immunoprecipitation was carried out by using control or specific antibodies coupled to Dynabeads (Invitrogen). The precipitated DNA fragments were purified and used for PCR analysis. Primers were designed for analysis of binding of DSG3 promoter: forward primer 5'-TCCGGACTAGCTTTTGCACT-3' and reverse primer 5'-GCTGCTTTCCGAAGGAGTCT-3'.

Passive transfer PV mouse model. Neonatal C57Bl/6J mice (The Jackson Laboratory, 2-3 days old, weighing between 1.2-2.5 g) were topically treated with DMSO, 5\% hydrocortisone, $12.5 \mathrm{nM}$ rapamycin, or 25 $\mu \mathrm{M}$ Stat3 inhibitor XVIII on the back for 3 days, followed by s.c. injection of $0.5-1 \mathrm{mg}$ PV IgG. Blister formation was evaluated at 16-18 hours; back skin samples were obtained for histopathology, and frozen sections were obtained for direct immunofluorescence analysis to detect autoantibody binding.

Statistics. Statistical analysis of DSG3 mRNA expression and cell sheet fragmentation was performed by 2-tailed Student's $t$ test or one-way ANOVA. Comparison of desmosome number and length was performed using Wilcoxon rank-sum test and $t$ test. Evaluation of gross blister formation in mice was performed using Kruskal-Wallis test with Dunn's post hoc multiple comparisons analysis. $P$ values less than 0.05 were considered significant. In all bar graphs, error bars indicate standard deviation. For blister histology scores, the mean is indicated by a horizontal line, with the $95 \%$ CI bracketed.

Study Approval. All studies were performed according to IRB and IACUC-approved protocols (University of Pennsylvania, Philadelphia, Pennsylvania, USA). A waiver of informed consent was obtained for human PV IgG samples, as these samples were not primarily collected for research purposes.

\section{Author contributions}

XM designed and conducted experiments, analyzed data, and wrote the manuscript. MJTC and CTE designed and conducted experiments, analyzed data, and reviewed the manuscript. EMM designed experiments, analyzed data, and reviewed the manuscript. ASP designed experiments, analyzed data, and wrote the manuscript.

\section{Acknowledgments}

Work in this report was supported by grant funding from the National Institute of Arthritis and Musculoskeletal and Skin Diseases to ASP (R56-AR064220), MJTC (F31-AR066456, T32-AR007465), and EMM (F30-AR065870, T32-AR007465) and from Deutsche Forschungsgemeinschaft to CTE (EL711/1-1). The contents are solely the responsibility of the authors and do not necessarily represent the official views of the NIH. We thank Tzvete Dentchev and Steve Prouty for IHC and histologic analyses, and Dewight Williams for electron microscopic analyses.

Address correspondence to: Aimee S. Payne, 421 Curie Boulevard, 1009 Biomedical Research Building, Philadelphia, Pennsylvania 19104. USA. Phone: 215.746.4488; E-mail: aimee.payne@uphs.upenn.edu.

1. Aoyama Y, Kitajima Y. Pemphigus vulgaris-IgG causes a rapid depletion of desmoglein 3 (Dsg3) from the Triton X-100 soluble pools, leading to the formation of Dsg3-depleted desmosomes in a human squamous carcinoma cell line, DJM-1 cells. J Invest Dermatol. 1999;112(1):67-71.

2. Calkins CC, et al. Desmoglein endocytosis and desmosome disassembly are coordinated responses to pemphigus autoantibodies. J Biol Chem. 2006;281(11):7623-7634. 
3. Mao X, Choi EJ, Payne AS. Disruption of desmosome assembly by monovalent human pemphigus vulgaris monoclonal antibodies. J Invest Dermatol. 2009;129(4):908-918.

4. Nguyen VT, Arredondo J, Chernyavsky AI, Kitajima Y, Pittelkow M, Grando SA. Pemphigus vulgaris IgG and methylprednisolone exhibit reciprocal effects on keratinocytes. J Biol Chem. 2004;279(3):2135-2146.

5. Grando SA, Laquer VT, Le HM. Sirolimus for acute pemphigus vulgaris: a case report and discussion of dualistic action providing for both immunosuppression and keratinocyte protection. J Am Acad Dermatol. 2011;65(3):684-686.

6. Silos SA, et al. Cloning of the gene for human pemphigus vulgaris antigen (desmoglein 3), a desmosomal cadherin. Characterization of the promoter region and identification of a keratinocyte-specific cis-element. J Biol Chem. 1996;271(29):17504-17511.

7. Yokogami K, Wakisaka S, Avruch J, Reeves SA. Serine phosphorylation and maximal activation of STAT3 during CNTF signaling is mediated by the rapamycin target mTOR. Curr Biol. 2000;10(1):47-50.

8. Zhang Z, Jones S, Hagood JS, Fuentes NL, Fuller GM. STAT3 acts as a co-activator of glucocorticoid receptor signaling. J Biol Chem. 1997;272(49):30607-30610.

9. Bromberg JF, et al. Stat3 as an oncogene. Cell. 1999;98(3):295-303.

10. Amagai M, Klaus-Kovtun V, Stanley JR. Autoantibodies against a novel epithelial cadherin in pemphigus vulgaris, a disease of cell adhesion. Cell. 1991;67(5):869-877.

11. Wada N, et al. Aire-dependent thymic expression of desmoglein 3, the autoantigen in pemphigus vulgaris, and its role in T-cell tolerance. J Invest Dermatol. 2011;131(2):410-417.

12. Akira S, et al. A nuclear factor for IL-6 expression (NF-IL6) is a member of a C/EBP family. EMBO J. 1990;9(6):1897-1906.

13. Vallania F, et al. Genome-wide discovery of functional transcription factor binding sites by comparative genomics: the case of Stat3. Proc Natl Acad Sci USA. 2009;106(13):5117-5122.

14. Hutchins AP, et al. Distinct transcriptional regulatory modules underlie STAT3's cell type-independent and cell type-specific functions. Nucleic Acids Res. 2013;41(4):2155-2170.

15. Hutchins AP, Diez D, Miranda-Saavedra D. Genomic and computational approaches to dissect the mechanisms of STAT3's universal and cell type-specific functions. JAKSTAT. 2013;2(4):e25097.

16. Wen Z, Zhong Z, Darnell JE. Maximal activation of transcription by Stat1 and Stat 3 requires both tyrosine and serine phosphorylation. Cell. 1995;82(2):241-250.

17. Decker T, Kovarik P. Serine phosphorylation of STATs. Oncogene. 2000;19(21):2628-2637.

18. Paulson M, Pisharody S, Pan L, Guadagno S, Mui AL, Levy DE. Stat protein transactivation domains recruit p300/CBP through widely divergent sequences. J Biol Chem. 1999;274(36):25343-25349.

19. Lee H, et al. Acetylated STAT3 is crucial for methylation of tumor-suppressor gene promoters and inhibition by resveratrol results in demethylation. Proc Natl Acad Sci USA. 2012;109(20):7765-7769.

20. Takeda K, et al. Targeted disruption of the mouse Stat3 gene leads to early embryonic lethality. Proc Natl Acad Sci USA. 1997;94(8):3801-3804.

21. Sano S, et al. Keratinocyte-specific ablation of Stat3 exhibits impaired skin remodeling, but does not affect skin morphogenesis. EMBO J. 1999;18(17):4657-4668.

22. Chan KS, et al. Disruption of Stat 3 reveals a critical role in both the initiation and the promotion stages of epithelial carcinogenesis. J Clin Invest. 2004;114(5):720-728.

23. Sano S, et al. Stat3 links activated keratinocytes and immunocytes required for development of psoriasis in a novel transgenic mouse model. Nat Med. 2005;11(1):43-49.

24. Chan KS, et al. Forced expression of a constitutively active form of Stat 3 in mouse epidermis enhances malignant progression of skin tumors induced by two-stage carcinogenesis. Oncogene. 2008;27(8):1087-1094.

25. Nappi O, Pettinato G, Wick MR. Adenoid (acantholytic) squamous cell carcinoma of the skin. J Cutan Pathol. 1989;16(3):114-121.

26. Griffin JR, Wriston CC, Peters MS, Lehman JS. Decreased expression of intercellular adhesion molecules in acantholytic squamous cell carcinoma compared with invasive well-differentiated squamous cell carcinoma of the skin. Am J Clin Pathol. 2013;139(4):442-447.

27. Jung AC, et al. A poor prognosis subtype of HNSCC is consistently observed across methylome, transcriptome, and miRNome analysis. Clin Cancer Res. 2013;19(15):4174-4184.

28. Khan K, Hardy R, Haq A, Ogunbiyi O, Morton D, Chidgey M. Desmocollin switching in colorectal cancer. Br J Cancer. 2006;95(10):1367-1370.

29. Saggar S, Zeichner JA, Brown TT, Phelps RG, Cohen SR. Kaposi's sarcoma resolves after sirolimus therapy in a patient with pemphigus vulgaris. Arch Dermatol. 2008;144(5):654-657.

30. Poot AM, Jonkman MF. Topical sirolimus for oral pemphigus vulgaris: 3 unresponsive cases. J Am Acad Dermatol. 2012;67(5):e228-e229.

31. Wong AL, et al. Phase I and biomarker study of OPB-51602, a novel signal transducer and activator of transcription (STAT) 3 inhibitor, in patients with refractory solid malignancies. Ann Oncol. 2015;26(5):998-1005.

32. Hong D, et al. AZD9150, a next-generation antisense oligonucleotide inhibitor of STAT3 with early evidence of clinical activity in lymphoma and lung cancer. Sci Transl Med. 2015;7(314):314ra185.

33. Sen M, et al. First-in-human trial of a STAT3 decoy oligonucleotide in head and neck tumors: implications for cancer therapy Cancer Discov. 2012;2(8):694-705.

34. Raney BJ, et al. ENCODE whole-genome data in the UCSC genome browser (2011 update). Nucleic Acids Res. 2011;39(Database issue):D871-D875 